\title{
A importância da construção, aplicação e ponderação dos sistemas de indicadores de sustentabilidade de forma participativa
}

Cícero Sousa Lacerda ${ }^{\mathbf{1}}$, Raquel de Lourdes de Miranda e Silva Carmona $^{1}$, Hercílio de Medeiros Sousa ${ }^{1}$, Josemary M. Freire Rodrigues de C. Rocha ${ }^{1}$, Érika Marques de Almeida Lima ${ }^{1}$, Patrícia Tavares de Lima ${ }^{1}$, Jaíze Costa do Nascimento ${ }^{1}$, Luciano Honório de Carvalho ${ }^{1}$, João Carlos de Miranda e Silva ${ }^{2}$, Natália Marques de Almeida Lima Miranda ${ }^{3}$

\footnotetext{
${ }^{1}$ Instituto de Ensino Superior da Paraíba - IESP. Estrada de Cabedelo, BR-230, $\mathrm{km} \mathrm{14,} \mathrm{s/n.} \mathrm{Morada} \mathrm{Nova.} \mathrm{Cabedelo-PB,} \mathrm{Brasil} \mathrm{(CEP} \mathrm{58109-303).} \mathrm{E-mail:}$ cicero@iesp.edu.br.

${ }^{2}$ Superintendência de Administração de Meio Ambiente - SUDEMA. Av. Monsenhor Walfredo Leal, 181. Tambiá. João Pessoa-PB, Brasil (CEP 58020-540).

${ }^{3}$ Instituto do Patrimônio Histórico e Artístico da Paraíba - IPHAEP. Av. João Machado, 348. Jaguaribe. João Pessoa-PB, Brasil. (CEP 58013-520).
}

Resumo. Para analisar a eficácia das ações propostas pelo modelo de desenvolvimento sustentável, posto como uma alternativa para um crescimento harmonioso entre o meio ambiente e a sociedade humana, surgem os modelos de indicadores de sustentabilidade. Estes modelos de indicadores apresentam diagnósticos da realidade atual e traçam perspectivas e estados desejados para o futuro, levando em conta os princípios da sustentabilidade. Neste sentido o objetivo deste artigo foi apresentar a importância dos sistemas de indicadores de sustentabilidade serem elaborados, aplicados e ponderados a partir da participação dos atores sociais locais. Para tanto utilizou-se o método da pesquisa bibliográfica por meio da leitura de artigos, dissertações e teses. Segundo a literatura pesquisada, observou-se que a utilização de sistemas de indicadores elaborados, aplicados e ponderados com a inclusão da sociedade local em todo o processo, os resultados terão mais confiabilidade, visto que a população local possui todos os conhecimentos com propriedade. Para tanto a utilização desses resultados participativos norteará melhor as tomadas de decisões o planejamento e o desenvolvimento local de forma sustentável.

Palavras-chave: Indicadores; Sustentabilidade; Construção de indicadores participativos.

Abstract. The importance of building, applying and pondering participatory sustainability indicator systems. To analyze the effectiveness of the actions proposed by a sustainable
Recebido:

$23 / 07 / 2019$

Aceito:

$30 / 08 / 2019$

Disponível on line:

$31 / 08 / 2019$

Publicado:

31/08/2019

Acesso aberto

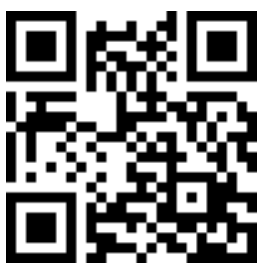

ORCID

(D) 0000-0002-4047-5540 Cícero Sousa Lacerda 
development model as an alternative to a harmonious growth between environment and human society, emerge the models of sustainability indicator. These indicator models present diagnoses of a current reality and outline desired perspectives and circumstances considering the principles of sustainability. Thereby, the objective of this paper is to present the importance of sustainability indicator systems being elaborated, applied and pondered from the participation of local social actors. For this purpose, we used a method on bibliographic research through articles, dissertations and thesis. In the light of the researched literature it was observed that the use of elaborated, applied and pondering indicator systems with the inclusion of the local society in the whole process, the results will be more reliable, since the local population has all the knowledge of the facts. To this end, the use of these participatory outcomes will better guide decision making in local planning and sustainable development.

Keywords: Indicators; Sustainability; Building Indicators.

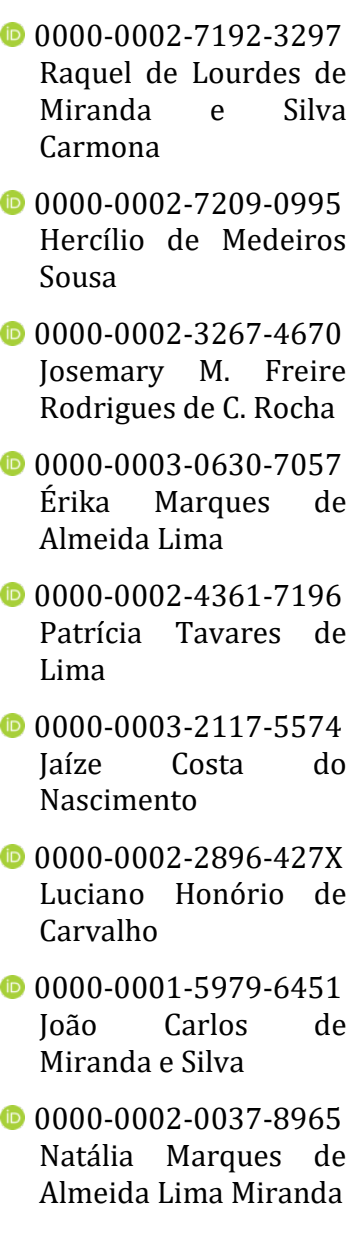

\section{Introdução}

Os sistemas de indicadores de sustentabilidade emergem como uma proposta de mensurar as ações do desenvolvimento sustentável. Nesse sentido esses indicadores aplicados a qualquer atividade econômica poderá analisar como encontra-se o seu estado diante a sustentabilidade. Porém se percebe que a maioria dos sistemas de indicadores são aplicados a partir de dados secundários que em sua maioria não apresenta respostas fidedignas do contexto atual. De acordo com a percepção de Silva et al. (2010) os indicadores de sustentabilidade são empregados como instrumento básicos em vários estudos nacionais $\mathrm{e}$ internacionais, promovendo a interpretação das informações sobre realidades complexas, e atua como apoio para mensurar o desenvolvimento que abrange as dimensões: ambientais, sociais, econômicas, culturais, geográficas, visto que facilita identificar os impactos negativo sobre o meio ambiente.

Para tanto compreende-se que a uma análise mais garantida sobre a aplicação de uma sistema de indicadores de sustentabilidade em um espaço geográfico ou em uma atividade econômica é necessário contemplar os atores sociais locais em todas as etapas do processo desde a identificação dos indicadores, a seleção até a ponderação dos mesmo. No contexto atual vários pesquisadores como Hanai (2009) e Cândido et al. (2010), apresentam a importância da utilização dos indicadores de sustentabilidade de forma participativa envolvendo a comunidade em todos os estágios desde a seleção, 
coleta e monitoramento dos indicadores. Pois compreende-se que os atores sociais são as pessoas que melhor respondem às particularidades presentes nas informações locais, bem como, precisam estar envolvidas no processo, sobretudo, por sofrerem os impactos negativos que atividade econômica poderá causar.

Os sistemas indicadores para envergarem fiabilidade, não podem ser elaborados a partir de especialistas políticos ou gestores que atuam sem contato com os usuários. Para melhor eficácia o mesmo deve ser elaborado em conjunto com os atores sociais incluindo os residentes na comunidade local que deverão estar envolvidos no processo de tomada de decisões relacionadas ao desenvolvimento e aplicação de indicadores de sustentabilidade (Cândido et al., 2010). Assim, a forma mais ideal para avaliar o nível de sustentabilidade de uma região ou atividade econômica é buscar uma visão holística dos efeitos causados em todas as dimensões que sofrem impactos com a atividade a atividade econômica como a dimensão ambiental, cultural, social, econômica, institucional e a própria dimensão ligada direto com as especificidades do contexto em análise.

Neste entendimento, é necessário que se tenha a participação da efetiva e envolvimento da sociedade local nos processos de planejamento e definição dos indicadores, como também nas possíveis tomadas de decisões com base nos resultados (Lacerda, 2011). Segundo Laura (2004), os indicadores de sustentabilidade devem contemplar a diversidade, portanto que sejam selecionados e definidos pela visão da sociedade, entendendo as características da comunidade, no intuito de torná-los aplicáveis e coerentes com a realidade local. Pois a utilização de indicadores como um instrumento de planejamento participativo colabora para uma maior conscientização, compromisso, além de dinamizar expectativas de aplicação das atuações conduzidas ao desenvolvimento sustentável local. Nessa concepção, “o desenvolvimento e aplicação de um sistema de indicadores de sustentabilidade consistente e participativo irá orientar as políticas públicas voltadas para a gestão do espaço ou da atividade econômica e dos recursos utilizados de forma sustentável" (Silva e Cândido, 2016).

Diante o exposto o objetivo dessa pesquisa consiste em apresentar a importância dos sistemas de indicadores de sustentabilidade serem elaborados, aplicados e ponderados a partir da participação dos atores sociais local. Para tanto se utilizou o método da pesquisa bibliográfica através de artigos, dissertações e teses. Utilizou ainda as bases de dados capes, scielo e bibliotecas virtuais universitárias.

A contribuição do trabalho pautou-se em orientar os trabalhos que utilizar os indicadores de sustentabilidade que inclua a participação dos atores sociais envolvidos no processo, mostrando que os mesmo possui informações importantes a direcionar o resultado da análise de forma mais assertiva. 0 planejamento e a gestão utilizando os indicadores de sustentabilidade deve ser participativo, como também as tomadas de decisão, dessa forma 0 desenvolvimento tende a ser mais equilibrado em atender as necessidades humanas bem como a sustentabilidade da dimensão ambiental.

\section{Sistemas de indicadores de sustentabilidade participativos}

Para elaboração e seleção dos indicadores de sustentabilidade no sentido de avaliar o nível de sustentabilidade de uma região ou comunidade é importante considerar as características do local. Segundo Zucarato (2006), os resultados das informações dos indicadores variam de acordo com as características de cada região e a necessidade de cada grupo social. Os indicadores quando selecionados em consonância com o 
contexto local que envolve a atividade econômica há uma maior possibilidade de ter uma avaliação mais fidedigna em relação ao nível de sustentabilidade da atividade. 0 desenvolvimento das atividades econômicas exige uma necessidade de ser avaliado e acompanhado para observar se o mesmo se encontra no caminho da sustentabilidade.

Segundo Silva et al. (2016), as atividades econômicas entendem que há uma necessidade prioritária de investigar cientificamente procedimentos e indicadores para mensurar as ações do seu desenvolvimento, e propiciar a elaboração de técnicas científicas para a sua aferição. Essa medição do nível de sustentabilidade das atividades econômicas contribui para auxiliar as tomadas de decisões e o gerenciamento sustentável de seu desenvolvimento. A utilização dos indicadores busca não apenas mensurar os impactos da atividade econômica, mas também identifica as estratégias e as ações que minimizam os prováveis conflitos negativos pertinentes ao seu desenvolvimento.

As avaliações do nível de sustentabilidade de uma atividade econômica, inicialmente só considerava a dimensão ambiental, porém como a atividade envolve todos os setores da economia e os aspectos de uma comunidade receptora. Posteriormente começa a perceber a importância de inserir indicadores sociais e econômicos para uma avaliação dentro do seu contexto, no sentido de obter uma análise mais ampla dos impactos da atividade. Acerca disso, Marnika et al. (2015) apresenta três classes de indicadores que interagem entre os pilares do desenvolvimento sustentável no sentido de mensurar os parâmetros de uma atividade econômica que podem afetar áreas protegidas.

0 uso de indicadores de sustentabilidade para o planejamento e desenvolvimento de uma região pode evitar impactos negativos ao meio ambiente. Bañon Gomis et al. (2011), abordam que alguns problemas ambientais estão ligados ao modo comum de agir. Nessa perspectiva as pessoas devem buscar evitar impactos negativos para meio ambiente, a sociedade e a economia, nesse sentido os indicadores de sustentabilidade podem alertar como obter uma relação de harmonia que proporcione uma vida promissora do desenvolvimento em todas as esferas.

Nesse contexto, os indicadores contribuem para o desenvolvimento humano, uma vez possibilita o homem refletir suas ações. Para Barter et al. (2012), os indicadores corroboram para a melhoria do crescimento humano, em nível de qualidade de vida humana e ambiental, pois os resultados estão ligados diretamente ao ser humano e no bem-estar. Geralmente observa-se que as atividades econômicas causam impactos positivos e negativos por se relacionar com várias outras atividades. Segundo Mowforth e Munt (1998), as atividades econômicas compreendidas como sustentáveis incentivam o desenvolvimento econômico, social, cultural e ambiental. Para tanto, é necessário que a seleção dos indicadores para analisar o seu nível de sustentabilidade contemple todas as dimensões.

0 desenvolvimento das regiões precisam ser avaliados constantemente e implementado estratégias que possa garantir sua continuidade de forma sustentável. Nessa perspectiva, Bluter (1999) considera que o ciclo de vida dos ecossistemas que não tiver uma avaliação continua de melhorias poderá inviabilizar a atividade a longo prazo. Pensar em um desenvolvimento a longo prazo é preocupar-se com a manutenção dos empreendimentos e a continuidade dos recursos. Nesse sentido, o uso de indicadores poderá auxiliar no planejamento a longo prazo das comunidades receptoras das atividade econômicas. Santos et al. (2018), apontam que os indicadores constituem parte fundamental para o planejamento e 
a gestão do desenvolvimento de uma região, proporcionando uma visão holística de todas as ações e dimensões com vista a continuidade dos recursos e a rentabilidade a longo prazo.

Os indicadores são uteis na elaboração de diagnóstico da realidade, deixando claro informações complexas e as relações do homem com toda atividade. Segundo Hanai e Espíndola (2010), os indicadores apontam os atributos relevantes de um sistema, deixando claro as complexas relações entre as diferentes variáveis envolvidas num fenômeno específico, tornando-o visível ou perceptível as informações contidas. Os autores ainda ressaltam que os indicadores são valiosos e úteis ferramentas para auxiliar nas tomadas de decisão em gestão, desenvolvimento e monitoramento de políticas de desenvolvimento sustentável. Além de serem utilizados em avaliação de nível global. Para Gallopín (1997, p. 1) são elementos necessários na avaliação global das melhorias com vistas ao desenvolvimento sustentável, pois os indicadores sugerem estados desejados para o futuro em todas as escalas global, regional e local.

Nesse sentido, os indicadores fornecem informações importantes para as tomadas de decisões assertivas. Afirma Meadows (1998) que os indicadores são itens que fornecem informações valiosas para a compreensão do todas global, para tomada de decisões e para o planejamento de estratégias de ações de melhorias. Além do simples processo de mensuração de dados, os indicadores incentivam a compreensão da relação das atividades econômicas com o meio ambiente e os seus impactos. Reed et al. (2006) reforça que, os indicadores de sustentabilidade podem impulsionar a percepção dos problemas sociais e ambientais, provocar a capacidade da comunidade em traçar ações e dirigir políticas e projetos de desenvolvimento sustentável.
Como é notório tem-se vários atividades econômicas que são impactante de forma negativa, se relacionando de forma ampla com todos aspectos de uma região é importante que seja realizado seu monitoramento contínuo para minimizar ações negativas ao meio. Segundo Hanai e Espíndola (2010) com o uso de indicadores é possível realizar o acompanhamento contínuo e consistente das transformações do desenvolvimento de uma atividade econômica ao longo do tempo numa comunidade. Os autores ainda ressaltam que os indicadores servem não apenas para informar sobre a situação do processo de desenvolvimento, mas permitem também verificar se os objetivos da sustentabilidade estão sendo atingidos.

A mensuração do desenvolvimento sustentável de uma atividade econômica ou de uma região deverá ocorrer de forma contínua em intervalos de tempo que possa ter um acompanhamento efetivo. Gallopín, (1997), concorda que, a mensuração da sustentabilidade não pode ser vista somente em um momento, mas sim como avaliação integrada ao longo do tempo para gerar informações, capazes de mostrar tendências, em diferentes escalas de tempo.

Os indicadores de sustentabilidade apresentam importantes parâmetro sobre a realidade das dimensões da sustentabilidade em relação as atividades econômicas. Segundo Silva et al. (2012), os indicadores de sustentabilidade são importantes parâmetros para apresentar a realidade, dentro de um contexto complexo, tendo como intenção fornecer informações necessárias para a tomada de decisão.

Para avaliar o desenvolvimento sustentável em diferentes escalas e espaços é indicado o uso de indicadores de sustentabilidade que sintetizem as informações. Herculano et al. (2008), apontam que os indicadores de sustentabilidade podem ser adotados como relevante ferramenta na avaliação 
do desenvolvimento sustentável em espaços de diferentes escalas, direcionando respostas que auxilia nas tomadas de decisões e implementação das políticas que propicie $\mathrm{o}$ desenvolvimento sustentável.

Diante o exposto, observa-se que as atividade econômicas poderão contribuir com o desenvolvimento local sustentável, porém é necessário que seja planejado de forma participativa levando em consideração os princípios da sustentabilidade e avaliado continuamente pelos indicadores de sustentabilidade, considerando as características locais e todas as dimensões que se relaciona com o contexto local.

\section{Considerações finais}

Os sistemas de indicadores de sustentabilidade apresentam como importantes ferramentas para análise do desenvolvimento sustentável de uma atividade econômica ou um espaço geográfico. Porém diante $\mathrm{o}$ contexto literário pesquisado observa-se que a construção dos sistemas de indicadores, a aplicação e a ponderação de forma participativa considerando os atores sociais local trará resultados mais positivos quanto ao nível de sustentabilidade de uma atividade econômica ou de uma região.

Os atores sociais são informados das características locais quanto aos aspectos sociais, ambientais, econômicos, culturais e das políticas públicas que tem apresentado como respostas aos problemas causados pelos impactos negativos das ações humanas. Assim considera a comunidade como fonte de informação importante e verdadeira quanto ao contexto local.

Também é importante a participação da comunidade nas tomadas de decisão, no planejamento e na gestão das ações do desenvolvimento local, a partir dos resultados dos indicadores de sustentabilidade. Os atores sociais precisam ser protagonistas de todo o processo do desenvolvimento sustentável. Pois são eles que sofrem com todos os impactos negativos advindos das ações do desenvolvimento regional.

Neste entendimento, o incentivo à efetiva participação dos atores sociais locais no processo de desenvolvimento local busca proporcionar a sua inclusão de maneira consciente e respeitosa na definição das ações desejáveis, buscando incorporar os princípios sustentáveis e valores éticos nas estratégias de planejamento e nos propósitos do desenvolvimento sustentável.

Nesta perspectiva, é necessário analisar o desenvolvimento das atividades econômicas a partir de um conjunto de indicadores que contemplem todas as dimensões da sustentabilidade que sofre influência das atividade econômicas utilizando métodos participativos, incluindo os atores sociais de todos os segmentos e entidades de classe local.

\section{Conflito de interesses}

Os autores declaram não haver conflitos de interesses.

\section{Referências}

Bañon Gomis, A. J.; Parra, M. G.; Hoffman, W. M.; Mcnulty, R. E. Rethinking the concept of sustainability. Business and Society Review, v. 116, n. 2, p.171-191, 2011. https://doi.org/10.1111/j.1467-8594.2011. 00381.x

Barter, N.; Russell, S. Sustainable Development: 1987 to 2012 - don't be naive, it's not about the Environment. Proceeding of the 11th Australasian Conference on Social and Environmental Accounting Research (A-CSEAR), University of Wollongong, 2012. p. 1-18.

Cândido, G. A.; Vasconcelos, A. C. F.; Sousa, E. G. Índice de Desenvolvimento Sustentável para o Município: uma proposta de metodologia com a participação de atores sociais e institucionais. In: Cândido, G. A. Desenvolvimento sustentável e sistemas de indicadores de sustentabilidade: formas de aplicação em contextos geográficos 
diversos e contingências específicas. Campina Grande: Ed. UFCG, 2010.

Gallopín, G. C. Indicators and their use: Information for decision making. In: Moldan, B.; Bilharz, S.; Matravers, R. (Eds.). Sustainability indicators: A report on the project on indicators of sustainable development. Chichester, GB: Wiley and Sons, 1997. p. 13-27.

Hanai, F. Y.; Espíndola, E. L. G. Indicadores de sustentabilidade: conceitos, tipologias e aplicação ao contexto do desenvolvimento turístico local. Anais do VIII Congreso Latinoamericano de Sociología Rural, Porto de Galinhas, 2010.

Hanai, F. Y. Sistema de indicadores de sustentabilidade: uma aplicação ao contexto de desenvolvimento do turismo na Região de Bueno Brandão, Estado de Minas Gerais, Brasil. São Carlos: Universidade Federal de São Carlos, 2009. (Tese de doutorado).

Herculano, F. C.; Lira, W. S.; Cândido, G. A. Índice de desenvolvimento sustentável no setor agrícola. Engenharia Ambiental, v. 5, n. 2. p. 14-23, 2008.

Lacerda, C. S. Sistema de indicadores de sustentabilidade para atividade turística: uma proposta metodológica participativa aplicada no Município do Conde/PB. Campina Grande: Universidade Federal de Campina Grande, 2011. (Dissertação de mestrado).

Laura, A. A. Um método de modelagem de um sistema de indicadores de sustentabilidade para gestão dos recursos hídricos-MISGERH: o caso da Bacia dos Sinos. Porto Alegre: Universidade Federal do Rio Grande do Sul, 2004. (Tese de doutorado).

Marnika, E.; Christodoulou, E.; Xenidis, A. Sustainable development indicators for mining sites in protected areas: tool development, Ranking and scoring of potential environmental impacts and assessment of management scenarios. Journal of Cleaner Production, v. 101, p. 59-70, 2015. https://doi.org/10.1016/ j.jclepro.2015.03.098
Meadows, D. Indicators and information systems for sustainable development: A report to the Balaton Group. Hartland: The Sustainability Institute, 1998.

Mowforth, A.; Munt, I. Tourism \& sustainability: new tourism in the thirdorld. London, UK: Routledge, 1998.

Reed, J. S.; Fraser, E. D. G.; Dougill, A. J. An adaptative learning process for developing and applying sustainability indicators with local communities. Ecological Economics, v. 59, p. 406-418, 2006. https://doi.org/ 10.1016/j.ecolecon.2005.11.008

Santos. J. G.; Cândido, G. A. Atividades turísticas e indicadores de sustentabilidade: um estudo em um destino turístico brasileiro. PASOS. Revista de Turismo y Patrimonio Cultural, v. 16, n. 1, p. 37-54, 2018.

Silva, A. M.; Correia, A. M. M., Cândido, G. A. Ecological Footprint Method: Avaliação da sustentabilidade no Município de João Pessoa, PB. In: Cândido, G. A. (Ed.). Desenvolvimento sustentável e sistemas de indicadores de sustentabilidade: formas de aplicações em contextos geográficos diversos e contingências específicas. Campina Grande: Ed. UFCG, 2010.

Silva, M. C.; Santos, J. C. V. Artesanato e cultura local: uma possibilidade de renda e desenvolvimento da atividade turística. Caminhos da Geografia, v. 17, n. 60, p. 31-47, 2012.

Silva, N. C.; Cândido, G. A. Sistema de indicadores de sostenibilidad del desarrollo del turismo: un estudio de caso del Município de Areia-PB. Revista Brasileira de Pesquisa em Turismo, v. 10, n. 3, p. 475-496, 2016. https://doi.org/10.7784/rbtur.v10i3.955

Zucarato, A. G.; Sansolo, D. G. Uso de indicadores na pesquisa em turismo. AIV SeminTUR - Seminário de Pesquisa em Turismo do Mercosul, 2006. 